\title{
Etnografía de la Planta Ballenera de Quintay: Reportajes, Informes, Historias
}

\author{
Ethnografy of Quintay Shore Whaling Station: Reports, Notes, Histories \\ Daniel Quirozi
}

\section{RESUMEN}

El procesamiento de una ballena en una planta costera terrestre comienza con la llegada de la ballena. La ballena era amarrada con una línea al huinche e izada a la rampa y luego a la plataforma de descuartizamiento. Allí se separaba el tocino, la carne y los huesos que iban a sus propias unidades de cocción o "cocinerías". Se obtenía aceite y una mezcla de líquidos y sólidos que eran enviados a plantas de purificación. Los residuos sólidos eran enviados a la planta de harina o de guano. En Chile se construyeron ocho plantas industriales costeras localizadas entre Punta Arenas por el sur e lquique por el norte. En este trabajo se intentará construir una etnografía retrospectiva de la planta ballenera de Quintay, utilizando los relatos en forma de reportajes, escritos por personas que observaron la planta en pleno funcionamiento. Nos interesa dar cuenta como han sido selectivamente representados determinados aspectos del procesamiento de las ballenas en una planta costera por aquellos que pudieron conocer las prácticas en un "presente etnográfico".

Palabras claves: Planta Ballenera, Etnografia Retrospectiva, Reportaje, Quintay.

\begin{abstract}
The processing of a whale in a shore whaling station began with their arrival. The whale was moored with a line to the winch and hoisted to the slipwayand on the platform flensing. At the platform,bubbler, meat and bones were separated and send to their own cooking units or "cocinerías". From cookeries, oil and a mixture of liquids and solids were sent to be purified. Solid wastes were sent to the whale-meal or guanoplant. Eight shore whaling station was built in Chile, located between Punta Arenas in the south and lquique in the north. In this paper, we construct a retrospective ethnography of Quintayshore whaling station,using the reports and written reports by people who observed the plant in full operation. In the reportages, the issues about whale processing, has been selectively represented by those who knew the practices in a "ethnographic present."
\end{abstract}

Key words: Shore Whaling Station, Retrospective Ethnography, Reportage, Quintay.

i Centro de Documentación de Bienes Patrimoniales, Dirección de Bibliotecas, Archivos y Museos. Recoleta 683, Santiago, Chile. Correo-e: Daniel.Quiroz@museosdibam.cl 


\section{INTRODUCCIÓN}

Se ha definido la caza de ballenas como "la matanza intencional de grandes cetáceos para obtener productos económicamente útiles” (Reeves y Smith 2006: 82). En la práctica, implica la realización de dos tareas separadas, la "matanza intencional" y la “obtención de productos económicamente útiles", es decir, la caza propiamente tal y el procesamiento de las carcasas de los cetáceos para obtener productos comercializables. Este trabajo se refiere más bien a esta última dimensión del problema.

La caza de ballenas con propósitos comerciales se inicia en el mundo occidental a partir del siglo $\mathrm{X} \circ \mathrm{XI}$ de nuestra era, cuando grupos de pescadores vascos capturan la ballena franca en el Golfo de Vizcaya para obtener la grasa, el aceite, la carne y las barbas que vendían por toda Europa (Azpiazu 2000). La caza moderna de ballenas, iniciada en los mares del norte de Noruega en la segunda mitad del siglo XIX se caracterizaban por usar "un arpón explosivo lanzado desde un cañón montado en la proa de un buque ballenero a vapor" (Brown 1976: 25). Los buques cazadores eran "pequeñas embarcaciones que parecen remolcadores, de 100 a 300 toneladas, de construcción fuerte, propulsadas a vapor y cuya tripulación era de unos once hombres" (Hohman 1935:632). El buque ideal debía ser "rápido, poderoso, maniobrable y virtualmente imposible de hundirse" (Davis et al. 1997:500). El hecho de contar con barcos rápidos permitió la captura de todo tipo de ballenas, incluidos los grandes rorcuales, como la ballena azul y la de aleta.

Las carcasas de los cetáceos eran procesadas en plantas terrestres para producir, en un comienzo, aceite y abono (Tønnesen y Johnsen 1982: 39-40). El proceso productivo desarrollado en una planta ballenera terrestre puede ser descrito, al mismo tiempo, como algo muy simple y muy complejo,

"era simple ya que hay unas pocas líneas de producción, que terminan en dos o tres productos principales, aceite y harina o guano de ballenas" pero era bastante complejo en sus detalles técnicos, representados por "numerosas y diversas cocinerías, estanques, separadores, válvulas, cintas transportadoras y tuberías, que iban en todas las direcciones" (Basberg 2004: 83).
Todo el proceso comenzaba con la llegada de la ballena a la planta. La ballena era amarrada con una línea al huinche e izada a la rampa y luego a la plataforma de descuartizamiento. Allí se separaba el tocino, la carne y los huesos que iban, cada uno, a sus propias unidades de cocción o "cocinerías". De las tres cocinerías se obtenía aceite y una mezcla de líquidos y sólidos que eran enviados a plantas de purificación. Los residuos sólidos eran enviados a la planta de harina o de guano (Basberg 2004: 83-84).

En Chile se construyeron ocho plantas industriales terrestres, destinadas al procesamiento de ballenas, localizadas entre Punta Arenas por el sur e lquique por el norte. La primera y la más meridional fue la de Bahía Águila, situada a $60 \mathrm{~km}$ al oeste de Punta Arenas. Esta planta fue construida en 1905 por la Sociedad De Bruyne, Andresen y Cia., empresa que dará origen en 1906 a la Sociedad Ballenera de Magallanes. En esa misma época, la Sociedad Ballenera y Pescadora de Valdivia levantará en 1906 la planta de San Carlos de Corral,Valdivia, y la Sociedad Ballenera Pacífico, una planta en 1909 en Isla San Pedro, al sur de Quellón, Chiloé. La Sociedad Pescadora Chile Noruega construirá entre 1921 y 1922 una planta en Caleta Samuel, Isla Huafo, al sur de la isla grande de Chiloé. En la década de 1930 la Sociedad Comercial Juan Macaya e Hijos construye una planta en Puerto Macaya, Isla Santa María, en el golfo de Arauco. En 1943 la Compañía Industrial S.A., inaugura su planta de Quintay, al sur de Valparaíso, y en 1956 la de Bajo Molle, al sur de Iquique. En I95I, la Sociedad Comercial Macaya Hnos. inaugura su planta de Chome, al sur de Talcahuano (Pastene y Quiroz 20 I0; Figura I).

La captura de ballenas en las costas de Chile es un "evento del pasado", por lo que su estudio requiere el uso de herramientas teóricas y metodológicas adecuadas, distintas a las tradicionales de la etnografía. El término "etnografía retrospectiva" nos sirve para nombrar un enfoque que involucra el uso integrado de diversos tipos de fuentes: recuerdos de personas que pudieron observar directamente la experiencia ballenera o les hablaron de ella; documentos administrativos, científicos y literarios, noticias y entrevistas de prensa; visitas a los lugares donde la caza de ballenas se produjo y relevamiento de sus testimonios y huellas materiales.

Charles Tilly (1978) define la etnografía retrospectiva como "el esfuerzo por reconstituir 


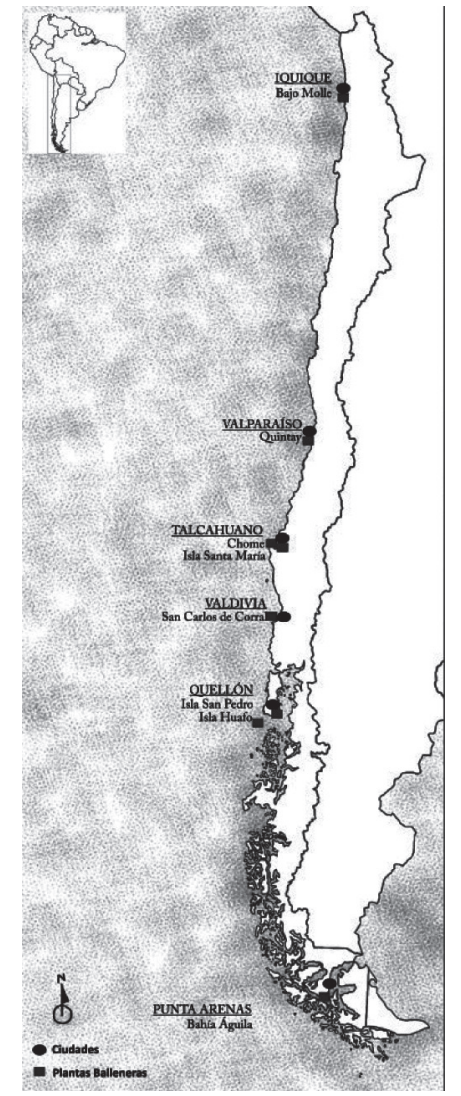

Figura 1: Mapa con la ubicación de las plantas balleneras en Chile [dibujo de Paula de la Fuente].

Figure 1: Map with the location of whaling stations in Chile [drawing Paula de la Fuente]

un modo de vida mediante los mejores equivalentes históricos de las observaciones de los etnógrafos, para luego usar el modo de vida reconstituido como contexto para la explicación de la acción colectiva". Los historiadores produjeron "historias" que pueden denominarse antropológicas pues "hacen hincapié en la reconstrucción de un modo de vida y de un cuerpo de significados desde la perspectiva de un observador participante en el campo" (Tilly 1978: 2 I0). Un ejemplo lo constituye la obra del historiador Keith Thomas, cuyo objetivo ha sido "construir una etnografía retrospectiva de la Inglaterra moderna temprana, abordando el pasado de la misma manera como un antropólogo aborda una sociedad exótica" (Thomas 2009:2). Una de sus líneas metodológicas ha sido la búsqueda de "miradas" y "voces" equivalentes a las que obtendríamos si existiera la posibilidad de observar la caza de ballenas y escuchar a sus practicantes en un "presente etnográfico", es decir, en ese momento definido por la co-presencia del etnógrafo y el "otro" en el trabajo de campo (Sanjek 1991: 609; Pina-Cabral 2000).

En este trabajo nos interesa desarrollar una etnografía de la planta procesadora de ballenas de Quintay, utilizando como fuente "primaria" de información los reportajes escritos por visitantes que estuvieron en la planta por distintos motivos, complementados con los recuerdos de personas que trabajaron en la planta ballenera.

\section{LA COMPAÑÍA INDUSTRIAL S.A.Y LA CAZA DE BALLENAS}

La Compañía Industrial S.A. se forma como sociedad anónima en Valparaíso a fines del900. El objetivo de la compañía era "la fabricación i elaboración de productos químicos, abonos artificiales, jabones e industrias anexas", para lo que adquieren la "fábrica de abonos artificiales, jabones i otros productos" que poseía la sociedad Anwandter \& Körner, en Carrascal, Santiago, la que debería constituir el punto de partida de sus negocios, un impulso para lo que sería un largo y exitoso desarrollo industrial (Indus 1938).

En el año 1935 la empresa decide extender sus actividades hacia la industria ballenera como parte de una política de autoabastecimiento que le permitiera proveerse de la materia prima necesaria para la elaboración de sus productos. Hasta ese momento la materia prima la había obtenido de otras compañías, principalmente de la Sociedad Ballenera de Corral, pero la producción nacional era insuficiente para satisfacer su alta demanda por lo que una parte muy relevante debía importarla. La escasez internacional de grasa obligó a la empresa a involucrarse en esta verdadera aventura ballenera (Salazar e Inostroza 1997: 73). Pese a que la caza de la ballena no era su principal rubro productivo, la INDUS logra transformarse en la empresa ballenera chilena más relevante de toda su larga historia.

Su primer movimiento fue la compra de los buques e instalaciones de la Sociedad Ballenera de Corral y de los buques de la Comunidad Chileno Noruega de Pesca (Pastene y Quiroz 2010). El 
interés original de la compañía era emprender operaciones pelágicas pues, después de "largas y profundas investigaciones en que han colaborado técnicos europeos de primer orden", concluyen que estas operaciones vendrán "a revolucionar todos los sistemas hasta ahora empleados en esta industria en el país" (Indus 1938: 7). Se compra y acondiciona el CÓNDOR, "uno de los vapores de mayor tonelaje en la Marina Mercante Nacional y que por sus características era el que ofrecía mayores condiciones para el objeto deseado" (Indus 1938: 7-8). De esta manera, la empresa se compromete con la caza pelágica y suspende la caza de ballenas desde las estaciones terrestres.

La irrupción de la Segunda Guerra Mundial detuvo momentáneamente la producción ballenera pues los países sumidos en la guerra entraron en una fase de inestabilidad de sus economías, dejando de funcionar gran parte de los mercados y paralizando las exportaciones de materias primas tales como el aceite de ballena. Las razones del abandono de la caza pelágica deben buscarse en el contexto de la producción ballenera internacional. Las empresas extranjeras estaban cazando ballenas en la zona antártica con buques-factoría mucho más grandes, modernos y poderosos. La competencia era, entonces, muy difícil. La compañía necesitaba realizar una seria inversión para instalar una planta terrestre que le permitiera dedicarse a la cacería en las costas del Pacífico, sin tener que competir con los buques fábricas internacionales (Quiroz y de la Fuente 20I2).

En la construcción de la nueva planta participaron unos 120 trabajadores, "entre los que destacaron mineros de la zona de Los Andes, encargados del movimiento de tierras por medio de tiros de dinamita y el trabajo de cantera para obtener la piedra adecuada en la construcción y muros de contención" (Salazar e Inostroza 1997: 76).

Este proceso de transformación del paisaje queda bien representado por las palabras de Roberto Montandón:

"un día llegaron los ingenieros y dinamitaron rocas en la punta que al sur remata la bahía. Levantaron edificios, agazapados al pie del farallón. Flanqueada de un muelle, una rampla bajó hasta el mar;una alta chimenea gris apuntó al cielo azul. Grupos de casas, enfrentando el océano, festonearon el acantilado allá en el alto; una estación de radio lanzó sobre su techo sus antenas verticales" (Montandón 1949: 8I).

\section{REPORTAJES}

La construcción de la planta y su posterior funcionamiento atraerá a una gran cantidad de visitantes, algunos de ellos escribirán sobre la experiencia. Algunos de estos textos adoptan la forma de "reportajes", ese género periodístico donde se mezcla información sobre hechos, o noticias, con opiniones personales del escritor, que buscan generar en el lector una posición sobre un tema específico. Gabriel García Márquez, un maestro en estos asuntos, nos dice que el reportaje puede "llegar a ser no sólo igual a la vida, sino más aún: mejor que la vida. Puede ser igual a un cuento o una novela con la única diferencia -sagrada e inviolablede que la novela y el cuento admiten la fantasía sin límites, pero el reportaje tiene que ser verdad hasta la última coma. Aunque nadie lo sepa ni lo crea" (García Márquez 200I).

\section{La inauguración de la planta ballenera}

La compañía invita a un grupo de periodistas en noviembre de 1943 a observar las faenas en la ballenera de Quintay, quienes entregan las primeras descripciones de la planta en funcionamiento. Uno de los relatos lo escribe G. Winet (1943), periodista de la revista Zigzag. Otro relato, más técnico y detallado, aparece publicado en forma anónima en la revista Mar, de la Liga Marítima de Chile (Anónimo 1943).

El relato de G. Winet nos dice:

"Los jefes de la industria nos llevan a visitar las diferentes instalaciones de la base: la gran rampa beneficiadora donde fácilmente puede caber cuatro ballenas, los grandes autoclaves extractores de aceite; la casa de fuerza, el salón de calderas, el pabellón de materiales. Todo realizado con los últimos adelantos de la técnica. A pesar de haber mucho construido, aún no está todo terminado. Siguen los trabajos. Actualmente se construye un estanque con capacidad para depositar seis mil toneladas de aceite [... Las instalaciones sociales] de la base ballenera [...], retén de carabineros, casa 
para los administradores, apartamentos para los empleados, casa para los obreros, policlínico, comedores, etc., están construidas en lo alto de la colina, más o menos unos 50 metros sobre el nivel de la playa propiamente dicha [...]. Después de recorrer las instalaciones levantadas para hacer más cómodo la vida del personal, pues doscientas personas trabajarán en esta base y de admirar las magníficas construcciones las casas para empleados y obreros dotadas de modernas toilettes, con baños fríos y calientes, excelentes dormitorios, amplias cocinas y panadería, etc. [...]. Ayudados por un buen catalejo divisamos en el horizonte marino un pequeño punto negro: es el INDUS III, que trae una ballena a cada costado [...]. La faena de descarga de la ballena es realmente interesante. El gigante de los mares viene atado de la cola por gruesas cadenas. Poderosos huinches arrastran luego la primera ballena por el plano inclinado, pequeño varadero construido especialmente en la base, hasta dejar el mamífero sobre la gran rampa beneficiadora, listo para ser abierto y despresado. Alrededor de una hora demoró la pasada faena. Los poderosos huinches tuvieron que emplear todas sus fuerzas para remolcar el cetáceo desde el barco hasta la gran explanada beneficiadora. La primera ballena medía 12 metros 50 centímetros. Y la segunda, catorce metros. Se calcula un peso medio de 50 toneladas por cetáceo [...]. La maniobra de remolcar las ballenas emplea todo los obreros de la base. Parecen hormigas en comparación con el volumen del gigante de océano"(Winet 1943: 15-16).

En el relato publicado en la revista Mar, el reportero nos cuenta:

"veíamos aparecer, como brotada de la tierra una planta industrial, en que no se ha omitido detalle alguno: desde el espléndido edificio para un retén de Carabineros, que se ve a la entrada del recinto industrial, hasta el pequeño departamento sanitario de clínica o enfermería para casos de primeros auxilios, sin que falten, por cierto, los departamentos destinados a las habitaciones para empleados y obreros, todos los cuales consultan las comodidades y el confort que ya quisieran para sí muchos de los que trabajan en las grandes ciudades para grandes empresas [...]. La planta está diseñada para una producción de unas 30 a 35 toneladas diarias de aceite, que se extrae por un procedimiento de cocción al vapor en grandes autoclaves de las grasas del animal, que las tiene en abundancia, y de la carne se extrae también aceite por un procedimiento al vacío, en un baño del propio aceite, aprovechándose el residuo de la carne, o sea, los chicharrones después de prensados en convertirlos en productos tales como abonos, alimentos para aves y animales, harinas, etc., de gran aplicación y consumo en el país y fuera de él. Además, es bien sabido que de los productos de la ballena se extraen también aceites de comer, jabones flotantes de muy buena calidad, margarina, esperma y otros [...] la energía eléctrica se obtiene de cuatro grupos electrógenos de 40 kilowatts, acoplados a motores diésel de petróleo crudo y la energía de vapor y el vapor mismo que se usa extensamente en la elaboración de los productos es proporcionado por dos calderas instaladas convenientemente en un pabellón especial que distribuye el vapor a los diferentes departamentos de la fábrica, devolviéndose el vapor ya gastado a una especie de condensador, para realimentar las calderas nuevamente [...]. Tanto las calderas de vapor como los grupos electrógenos son alimentados con petróleo crudo [...]. A las 5 de la tarde [... llegó el ballenero, al que] se veían amarrados a sus costados dos grandes bultos negros y en un momento como para sacar a todos de las dudas de dejaron oír dos pitazos con los cuales el INDUS III indicaba que regresaba a la base conduciendo dos grandes presas arrebatadas al mar. Inmediatamente se tomaron en la planta las disposiciones para colocar en tierra los enormes mastodontes y llegado [...] el buque a una distancia de 100 metros del muelle, se amarraron por la cola a un grueso cable de acero y con el auxilio de un huinche de 40 toneladas se procedió a subirlas suavemente por un plano inclinado de concreto construido ex profeso hasta colocarlas en una gran meseta de madera que actúa de mesa de operaciones en donde se despedaza el animal para su beneficio. Así quedaron colocadas una al lado de la otra y listas para empezar su beneficio" (anónimo 1943: 2 II-2 I2). 
Estos relatos subrayan el "prodigio tecnológico" que significa la construcción de la planta ballenera de Quintay. Describen sus principales instalaciones y señalan lo que queda todavía por desarrollar. Luego observan el traslado de dos ballenas desde el buque cazador hasta la plataforma de descuartizamiento, pero no son testigos de su procesamiento, debido, tal vez, a lo corta de su visita. En definitiva, es la infraestructura de la planta lo que se resalta en estos reportajes (Figura 2).

\section{Los años de gloria: la década de los 50}

La década de los 50 es la época de mayor auge de la industria ballenera chilena. El interés de diversos medios de prensa, no solamente del país sino del extranjero, por hablar de sus logros es un buen ejemplo de ello. Escogimos dos textos para ejemplificar este interés, uno escrito en una revista para "hombres" y el otro en una revista para "mujeres"

En 1953 la revista "para hombres" Male publica un extenso reportaje cuyo objetivo es mostrar la caza de ballena. Aunque el texto (anónimo 1953) nos habla principalmente de la cacería, entrega algunos datos de interés sobre el funcionamiento de la planta. El buque cazador en el que iba a bordo traía tres ballenas,
"Tuvimos que esperar nuestro turno en los muelles. Había 2 I ballenas antes de las nuestras para procesarlas. Todavía estaba enfermo. No sé si alguna vez estuve tan enfermo en mi vida. El olor de los restos de las ballenas es el peor negocio en el mundo. Pero había venido de tan lejos, tenía mi máquina fotográfica, y no iba a desaparecer hasta que ver el término de las operaciones. La primera ballena fue arrastrada a la plataforma de descuartizamiento del enorme muelle de la planta en la orilla. Esperamos en línea hasta que los trabajadores con cuchillos de mango largo se acercaron y comenzaron a cortar las capas externas de carne. Luego, la piel fue enganchada con un cable y una pinza a un huinche y la ballena fue pelada como si fuera la cáscara de un pomelo. Cada vez que se movía la ballena, 50 galones o más de sangre corrían sobre el cemento. De lo que sucedió después no entraré en detalles. Fue lo peor de todo [...]. Bajé cuando la primera ballena estaba siendo cortada. Cuando llegué, ya estaban en la tercera y yo, listo para ir a casa. Pero para llegar tuve que caminar otra vez a través de un caos sanguinoliento. Antes de que pudiera alcanzar el jeep de la oficina, me caí de nuevo -como dos días antes-y quedé rojo y hediondo. Llegué al jeep, que por ahora era verde con ruedas rojas, y cuando estaba

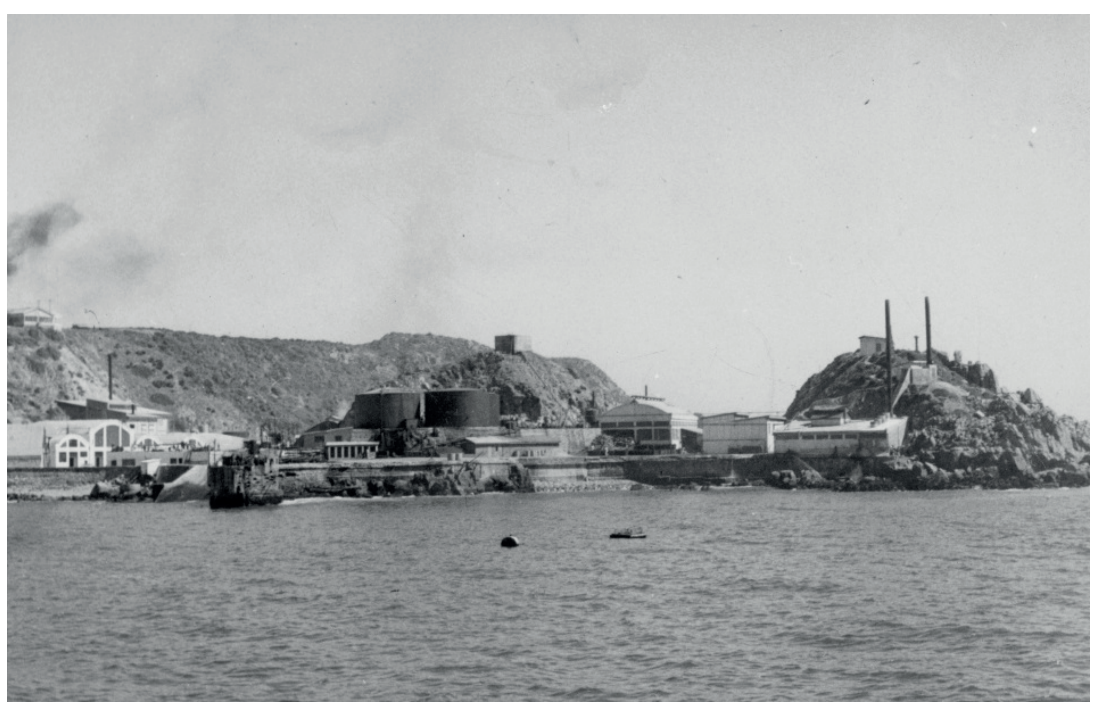

Figura 2: Vista de la planta ballenera de Quintay [Foto Archivo Iconográfico Museo Histórico Nacional]. Figure 2: View of the Quintay whaling station [Photo Archivo Iconográfico Museo Histórico Nacional]. 
tomando fotos de las operaciones de corte de la ballena más grande, el capataz se resbaló y cayó al suelo. Me sentí muy bien [...] y comencé a reír. Pero no fue gracioso. El chico se rompió la pierna" (anónimo 1953: 42-43).

Otro reportaje interesante y novedoso, escrito por Heinz Tischer (1957) fue publicado en la revista alemana Constanze. El texto se inicia con una referencia a la Compañía Industrial S.A., "una poderosa sociedad industrial [...que] posee nueve fábricas en todo el país, entre ellas la ballenera" de Quintay. Indica que si se revisa su lista de directores, se "tiene la impresión de haber aterrizado en una empresa alemana: Heinsen, Gaudnitz, Appuhn, Stelzner, Brüggemann, Schlenker, Ziegler $-y$ así suma y sigue". Inclusive "el director de pesca era un alemán, se llamaba Capitán Niemeyer”. El capitán Niemeyer, "antiguo comandante de submarinos y más tarde oficial de la Flota de Onassis" había sido contratado "en Delmenhorst, cerca de Bremen, para que reviviera la casi ya muerta captura de ballenas". El reportero observa, desde lo alto de un cerro, "la fábrica, a orillas del mar". Un buque cazador "había traído, en la noche, dos ballenas". Una de ellas, "un cachalote de 15 metros de largo se encontraba tendido sobre la cubierta de faenamiento, la segunda recién la estaban sacando con cuerdas del agua, por una pista de cemento con pendiente". Le impresiona de sobremanera el cachalote,

"Nunca me hubiera podido imaginar tan vivamente un cachalote. No parece haber alguna foto que lo muestre en su totalidad, junto a una persona. Moby Dick, su monumento literario, había sido imitado poderosamente para la película. Pero yo quería ver Moby Dick al natural y tomarle una foto realmente ilustrativa. ¡Y ahí está un poco más adelante! Me costó un par de zapatos. Quiero decir: los zapatos, después de haber caminado con ellos sobre aceite, sangre y aceite de pescado, quedaron resistentes al agua por toda una eternidad, pero por la hediondez a aceite de pescado, utilizables únicamente para labores domésticas. El cachalote pesa cincuenta mil kilos. Su colega, la ballena azul (una ballena con barbas, mientras que el cachalote en vez de barbas tiene dientes) es más pesada. Pero la ballena azul es más rara de encontrar en la corriente de Humboldt de la costa sudamericana, y no es ni de lejos tan aventurera. Los trabajadores,

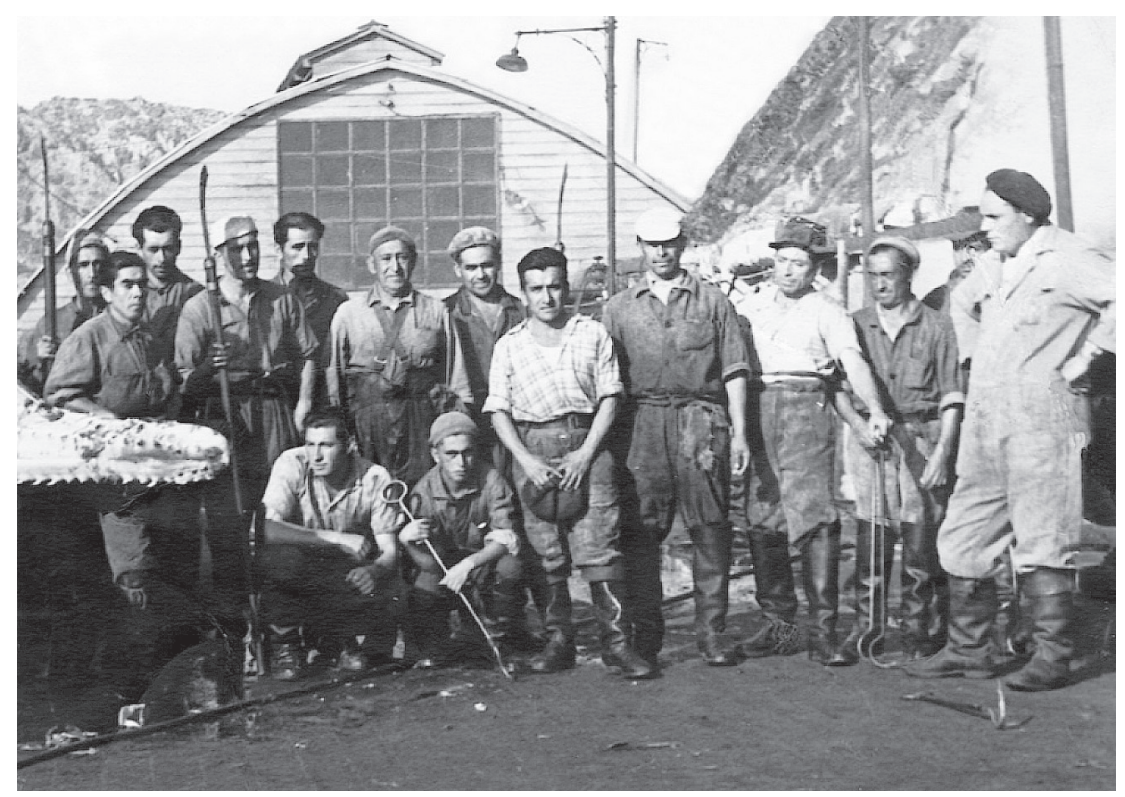

Figura 3: Uno de los turnos de la Sección de Descuartizamiento, donde se observan cuchillos y ganchos para el procesamiento de la ballena, c. 1960 [Foto Archivo Iconográfico del Museo Histórico y Antropológico de Casablanca].

Figure 3: Flensing Section, where knives and hooks for whale processing are observed. c. 1960 [Photo Archivo Iconográfico del Museo Histórico y Antropológico de Casablanca]. 
cuando quieren subir, cortan escalones en el cerro de carne y grasa" (Tischer 1957:93).

Finalmente señala que:

"muy pronto se llegará a la ballena 12.000 desde la fundación de Quintay en 1944, que se procesará como aceite, margarina, jabón y harina para animales. Toda la harina que se hace de la carne durante todo un año está vendida a Alemania, donde sirve como alimento fuerte para el aumento de grasa de nuestro querido ganado y para que nuestras gallinas pongan huevos" (Tischer 1957: 94).

Estos dos relatos son escritos por reporteros extranjeros. Ambos pueden contemplar el procesamiento de los cetáceos e indicar el impacto que significa su "carnicería", retratar la sangrienta plataforma de descuartizamiento y el mal olor que inunda las faenas. Existen algunas diferencias en el enfoque debido, probablemente, al público de sus revistas, una para hombres, donde se subraya el peligro que corren los trabajadores, y la otra para mujeres, donde es notable la importancia que se le da al par de zapatos estropeados.

\section{El ocaso: la década de los 60}

La década de los 60 es una época de decaimiento de la actividad ballenera en Quintay.
En 1966 se publica en El Mercurio de Valparaíso un nuevo reportaje sobre la planta, escrito por Gastón Gauche (1966). Este texto está centrado en la caza de la ballena, pero también entrega información importante sobre el procesamiento de los cetáceos,

"Al llegar el cazador a la caleta en la cual está ubicada la planta, una pequeña embarcación sale a recibirla.A su popa amarra los dos cetáceos traídos y vuelve a tierra [...]. En la orilla se levanta una ancha rampa encementada. Unos pescantes cogen las ballenas por los muñones de sus colas y comienzan a tirar. Con crujidos de cadenas el enorme cuerpo comienza a ser sacado del mar. Poco a poco sale toda la inmensa masa [...]. Una vez colocada en la rampa un grupo de hombres se abalanza sobre este enorme cuerpo y con diestros cortes comienzan a faenarla". El reportero establece algunas diferencias en el procesamiento de ballenas de dientes y de barbas. "Se desprende en primer término la envoltura o capa de grasa que la recubre, llamada 'tocino'. Ella es conducida a unos inmensos cocinadores $y$ arrojada adentro. Luego se da vuelta el cuerpo y se le extrae la capa inferior. Queda el esqueleto con un poco de carne y sosteniendo todos los enormes órganos del cetáceo, y la cabeza que requiere de una faena especial. Nuevos cortes ponen al descubierto una masa de tejidos

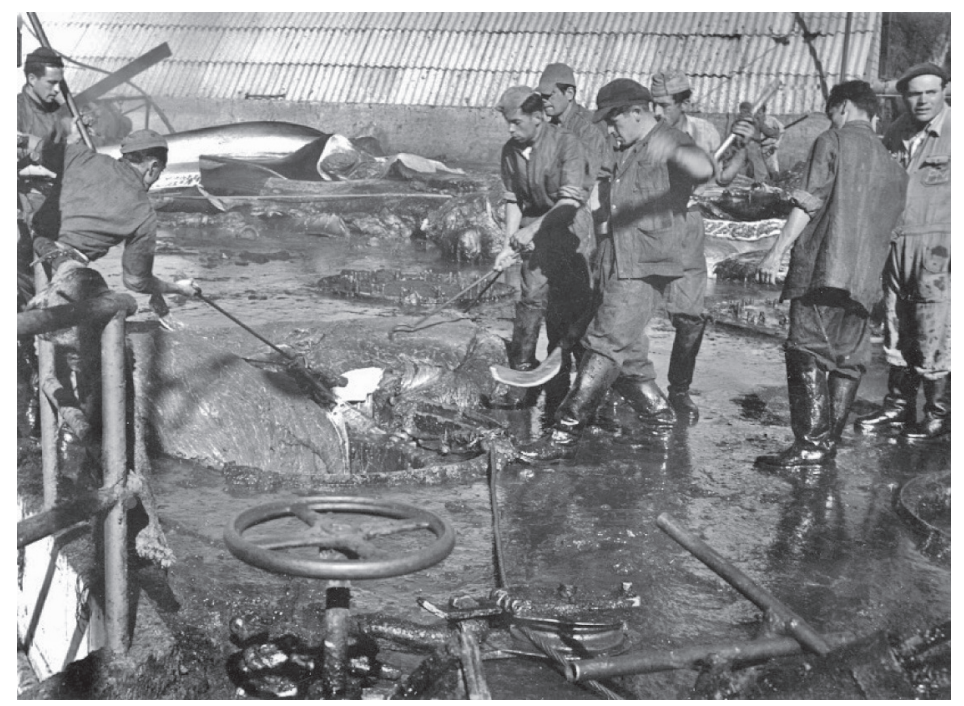

Figura 4: Lanzamiento de trozos de tocino a los autoclaves. Se observan cuchillos y ganchos, c. 1960 [Foto gentileza de Juana Álvarez, Quintay].

Figure 4: Pieces of blubber to the autoclaves. Knives and hooks are observed, c. 1960 [Photo courtesy of Juana Alvarez, Quintay]. 
ricos en aceite, los que son también llevados al cocinador donde está el tocino. Mientras tanto otros operarios cortan el esqueleto y se arroja a un cocinador que dará aceites de segunda clase. En sólo media hora la ballena ha desaparecido de la rampa. Sólo unos charcos que son limpiados con un fuerte pistón señalan el lugar de trabajo" (Gauche 1966: I5).

Esta descripción se refiere al procesamiento del cachalote y a la elaboración del aceite.

En el caso de la ballena azul o alfaguara y de otras ballenas de barbas el proceso tiene algunas diferencias.

"Las grandes lonjas de grasa y carne que la recubren son llevadas íntegramente hasta el barco frigorífico, donde cortadas y envasadas en bolsas de polietileno, son transportadas al Japón, país que se alimenta en gran parte con la ballena. El enorme esqueleto de una ballena del tipo barba, presenta adherido grandes trozos de carne, los que son cuidadosamente extraídos y llevados al frigorífico. Su lengua, riñones, corazón, hígado y otros órganos son sacados cuidadosamente y frigorizados para ser luego consumidos. Posteriormente el esqueleto es cortado por una sierra y arrojado a un cocinador para aceite. Otra vez, del magnífico animal no ha quedado nada" (Gauche 1966: I5).
Luego de recibir los trozos de tocino, carne y huesos,

"se cierran los cocinadores y se echan a andar por cuatro horas. Al abrirlos se tendrá un aceite que será destinado a diversos usos. Los del primer cocinador darán posteriormente margarinas, cosméticos y otros productos industriales. Los aceites de segunda se convertirán en jabones de menor calidad o serán tratados nuevamente hasta convertirse en harina, utilizada para alimentos de aves $y$ para abonos agrícolas" (Gauche 1966: I5).

El reportero agrega que:

"los trozos de carne de la ballena serán envasados y vendidos en el extranjero, porque un país como el nuestro aún no ha comprendido [...] que la mejor fuente de alimentación la tiene en sus aguas. La carne es, a simple vista, de la misma naturaleza que la carne de vacuno. Sólo su color es levemente más oscuro y presenta, al comerla, el mismo gusto que la que habitualmente se consume" (Gauche 1966: I5).

Finalmente, "el agua que arroja una manguera elimina todo vestigio de la ballena, y junto con su escurrir se va la última visión de esta aventura del mar y de este esfuerzo titánico de la industria" (Gauche 1966: I5).

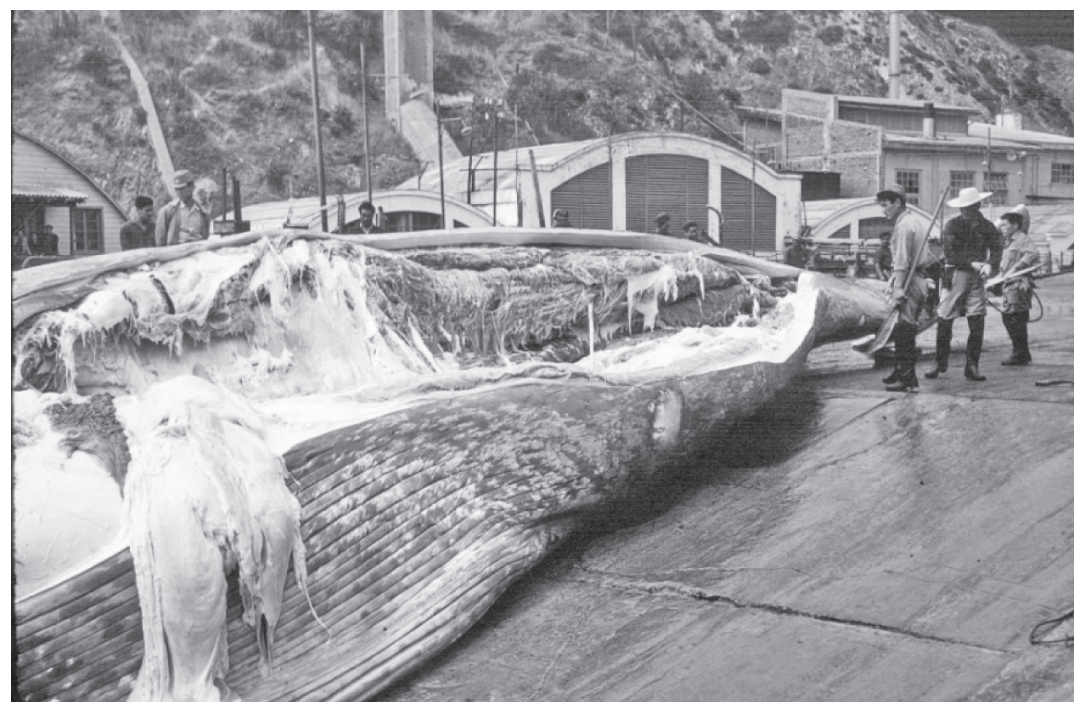

Figura 5: Descuartizamiento de la ballena para la producción de carne con trabajadores japoneses, c. 1965 [Foto Universidad Andrés Bello-CIMARQ].

Figure 5: Whale's flensing for meat production with Japanese workers. C. 1965 [Photo Universidad Andrés Bello-CIMARQ]. 
Este relato es fiel testimonio de una transformación ocurrida en la planta de Quintay con la llegada en 1964 de la empresa japonesa Nitto Hogei KK y su alianza con la Compañía Industrial S.A., que opera entre 1964 y 1967. El principal objetivo de las instalaciones balleneras era ahora la producción de carne para consumo humano, mayoritariamente exportada a Japón, pero también consumida en hogares chilenos (Figura 5). El texto muestra las diferencias observadas en el procesamiento de las ballenas de dientes y la producción de aceite y las ballenas de barbas y la producción de carne.

\section{RECUERDOS}

Es importante complementar la información proporcionada en los reportajes con los recuerdos de aquellos que participaron, directa o indirectamente, en sus faenas. La planta estaba formada por diversas construcciones, siendo la más conspicua, sin duda, su plataforma de descuartizamiento, que "la hace visualmente diferente de cualquier otra planta industrial" y constituye el verdadero núcleo de las instalaciones (Basberg 2004: 86). El espacio que tenemos nos impide abordar la planta por completo, así es que sólo nos referiremos a la Plataforma de Descuartizamiento (Figura 6).
La Plataforma de Descuartizamiento era una estructura semi-rectangular de concreto, de unos $50 \times 25 \mathrm{~m}$, con una cubierta de madera, unida a una rampa, un plano inclinado que llegaba hasta el agua. A un costado de la rampa estaba el muelle. Los barcos cazadores dejaban las ballenas en una boya, cerca de la costa, y regresaban al mar para continuar cazando.

Isaías Jaramillo indica las ballenas eran remolcadas desde las boyas al muelle de la planta ballenera en unas pequeñas embarcaciones llamadas pangas: "había unos botes, unas pangas que se llamaba, [...las ballenas] que estaban en las boyas había que traerlas al muelle en las pangas y con temporal iban a buscarlas igual, los botes parecían de papel encima del agua y las acarreaban igual" (Isaías Jaramillo, Quintay, 2008). En el muelle estaba el donkey, una pequeña grúa que era utilizada para izar las ballenas a la rampla. José recordaba que,

"antes amarraban las colas de las ballenas sólo con cabos metálicos, sin embargo, así se cortaban las colas de la ballena y éstas caían nuevamente al mar, y tenían que hacer una tremenda maniobra pa' sacarla [...] después mandaron a hacer la jaibay, dio tanto resultado que nunca se les cortó la cola, y donde la agarraba, mientras más tiraba más apretaba" (José Barrios, Quintay, 2008).

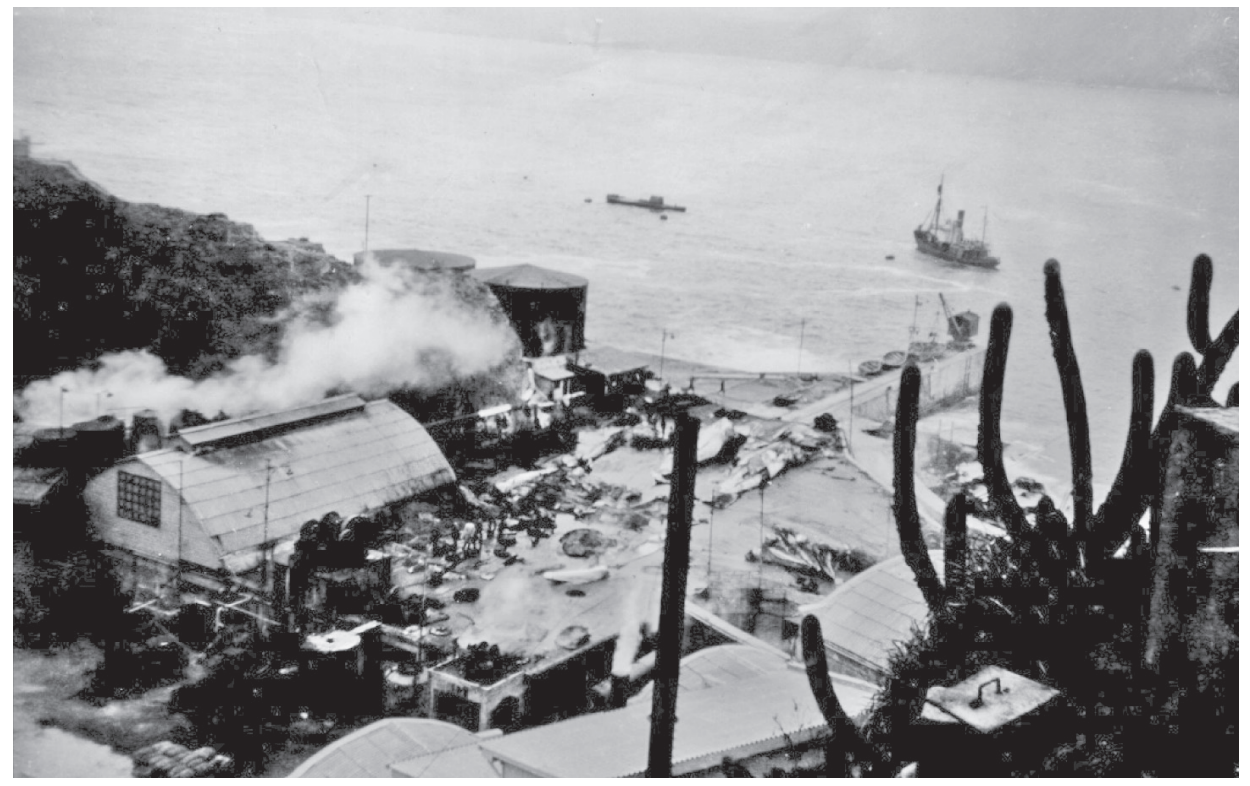

Figura 6: Plataforma de descuartizamiento, c. 1950 [Foto gentileza de Héctor Mendieta].

Figure 6: Flensing platform, c. 1950 [Photo courtesy Héctor Mendieta] 
Isaías agrega que para llevar la ballena a la plataforma de descuartizamiento se usaba un huinche grande, situado en la parte posterior de la plataforma.

"Este tiraba un cable, un cable guía, una línea, que llegaba hasta el muelle donde lo pescaba el donkey que tenía una tremenda tenaza,jaiba que le decían, y esa la metían en la cola de la ballena [...] con ese [huinche] tiraba la ballena hasta llegar arriba [...] y los huinches chicos, en los lados de la cama de descuartizamiento, con esos trataban que la línea no se moviera, no se tumbara para los lados" (Isaías Jaramillo, Villa Alemana, 20 (0).

José nos cuenta que "deben haber habido por lo menos unos diez huinches, porque en todas partes había huinches, estaban pa' tirar, tiraban pa'llá, tiraban pa'cá, sacaban pa' todos lados [...], con dos huinches daban vuelta la ballena y con otro le sacaban el cuero" (José Barrios, Quintay, 2008). Su hermano, Daniel Barrios, agrega que,

"Cuando le sacaban el cuero por una parte, después pescaban la ballena, hacían una maniobra y la daban vuelta, con los huinches empezaban a virar, a virar, y la daban vuelta pa'l otro lado y le sacaban la otra parte de cuero por el otro lado, después le iban sacando los lomos, después las tripas, después las costillas, iban sacando con los cuchillos las costillas, iban saliendo las costillas enteritas, se cortaban así, un corte no más, y se iban echando a los cocinadores" (Daniel Barrios, Quintay, 2008).

Para descuartizar a los cetáceos se usan unos cuchillos curvos, que eran como "una echona, esa pa' cortar trigo [...], pero con el filo por fuera, porque uno al trabajar va cortando la carne con la espalda del cuchillo" (Alfredo Marín, Quintay 2009). El faenamiento de una ballena era rápido, duraba entre cuatro y ocho horas (Berríos 20l0).

Los cocinadores o autoclaves estaban en un extremo de la Plataforma de Descuartizamiento, "por debajo, y la pura boca estaba aquí arriba [...] por la boca nosotros les tirábamos pa' abajo la carne [...] hasta que llenábamos ese autoclave, que era más o menos de unos cuatro o cinco metros de alto y tenía unos cuatro o cinco metros por lo menos de circunferencia" (Alfredo Marín, Quintay, 2009). José recuerda que para sacar el tocino usaban un gancho "el gancho era de un fierro que estaba arqueado en la punta [...], el tocino es una tira larga, entonces la iban cortando con los cuchillos en pedazos cuadrados y eso lo iban tirando, con esos ganchos lo iban tirando adentro pa' cocinarlos" (José Barrios, Quintay, 2009). Debajo de la plataforma se encontraba la denominada Fábrica de Aceite.

De este modo, la sección de descuartizamiento se encargaba de la ballena desde que era dejada en la boya por los buques cazadores hasta que sus restos eran depositados en los cocinadores o autoclaves (Figura 4). Se operaba con tres turnos de ocho horas cada uno, encabezada por un Jefe de Sección o Capataz, del que dependía una verdadera jerarquía laboral: maestro descuartizador, ayudante de maestro, ganchero y alambrero. En cada turno trabajaban dos 0 tres maestros descuartizadores, cada uno con su ayudante, tres o cuatro gancheros y tres alambreros (de la Fuente y Quiroz 20lI). Los maestros eran los responsables de descuartizar la ballena. Tenían que conocer la anatomía de la ballena para hacer los cortes.

"Conocerle todas las coyunturas, cómo cortar las coyunturas, porque los huesos son gruesos [...] dónde y cómo cortar los lomos y las costillas, hasta que queda la pura cabeza con el espinazo hacia un lado [...] mirando yo lo aprendí y les enseñamos con mi hermano a otras personas que vinieron de lquique $a$ trabajar. Los maestros conocían los cortes [...] para sacar el tocino y así pelar el animal" (José Barrios, Quintay, 2008).

Los ayudantes asistían al maestro en los cortes, dando instrucciones a los que operaban los huinches. Los alambreros se ocupaban de los alambres, los cabos de metal de los huinches que se usaban "para pelar la ballena y darla vuelta". Los gancheros llevaban los trozos de tocino, carne, vísceras y huesos a los cocinadores (de la Fuente y Quiroz 20I I).

\section{CONCLUSIONES}

La información contenida en los reportajes contribuye, sin duda, a comprender lo que ocurre en la plataforma de descuartizamiento de la planta cuando llega una ballena. Desafortunadamente, no hablan mucho de las personas involucradas en la caza 
y procesamiento de las ballenas. Sus descripciones apuntan a subrayar un aspecto "heroico" de la actividad ballenera, la caza propiamente tal. Las narraciones referidas a la planta recogen el lado más bien "prosaico", conformado por elementos tales como descuartizamientos, "malos olores" y "restos sanguinolentos" entre otros temas que podemos etiquetar como "gore". Este es un aspecto presente en prácticamente todas las plantas balleneras en el mundo. Recurrir a los recuerdos nos permite llenar un poco los vacíos que dejan los reportajes. Los testimonios son vivenciales, trasmiten aspectos más cotidianos del procesamiento de las ballenas, la estructura de trabajo existente y de los distintos roles que las personas desempeñaron. En este sentido, los reportajes y los recuerdos se complementan.

Pero también creemos necesario introducir una mirada más técnica, como la que se entrega en un informe publicado en Norsk Hvalfangst-Tidende, revista noruega especializada en temas balleneros. Es un relato que no deja mucho lugar a las emociones,

"En la planta de Quintay el trabajo es desarrollado por tres turnos, cada uno de ocho horas, así siempre hay un equipo listo para recoger las ballenas en las boyas, subirlas a la plataforma de descuartizamiento y comenzar con la extracción y el procesamiento en la forma habitual de la grasa, carne, huesos, etc. El procesamiento es efectuado en las distintas instalaciones, con las más modernas maquinarias importadas de Europa y otras diseñadas y hechas en Chile, sobre la base de la experiencia acumulada durante muchos años. Parte de la carne de la ballena de aleta es usada en estado fresco para el consumo humano en Valparaíso y Santiago, el resto es secado y convertido en comida para aves y para la exportación. Parte del aceite de ballena es hidrogenizado y usado en la manufactura de margarina y de tipos especiales de jabones, incluido el jabón de tocador. Los huesos son sometidos a un proceso de extracción con solventes de los materiales grasos separados para usarlos en la elaboración de jabones. Luego los someten a otro proceso para la extracción de la cola, que se vende en forma de tabletas para el uso de los carpinteros, tanto en país como en el extranjero. El resto de los huesos es secado y usado como fertilizante en el sur del país. De esta manera las ballenas son usadas completamente, incluso el agua de la cola es centrifugada para recuperar el último pedazo de sustancia útil. La industria ballenera en la costa de Chile es una industria completamente chilena, con respecto tanto al capital como a la operación [...] el personal de la planta, incluidos los ingenieros y químicos, son chilenos. Todas estas personas, en cincuenta años de actividades balleneras, se han familiarizado con las operaciones balleneras" (Cl 1954: I77).

Este informe permite cerrar la etnografía de la planta ballenera en términos del aprovechamiento de cada una de sus partes.

Para terminar, es importante recordar que en el mes de abril de 1953 el escritor Francisco Coloane visita Quintay para participar en una cacería de ballenas a bordo del Indus II, buque de la flota ballenera de Indus. Los datos recogidos en esa oportunidad le servirán para escribir un largo reportaje denominado Los balleneros de Quintay, publicado cuando la caza de ballenas en ese lugar era solo un recuerdo (Coloane 1972). Transformado en cuento, el relato finaliza con la siguiente frase: "No he regresado a Quintay porque hoy es un lugar desolado, sin cachalotes ni balleneros. Solo la trompa del cerro Curauma se asoma en la playa oceánica con el grito universal del Green Peace" (Coloane 2002: 305).

Agradecimientos: La información utilizada para escribir este trabajo fue reunida en el marco del proyecto Fondecyt I I I 082 Antropología e historia de la industria ballenera en Chile (1936-1983). Mis reconocimientos a Paula de la Fuente y Violeta Berríos por sus comentarios a versiones previas de este trabajo.

\section{BIBLIOGRAFÍA}

Azpiazu, J. A. 2000. Balleneros Vascos en el Cantábrico. Ttarttalo, San Sebastián.

Basberg, B. L. 2004. The Shore Whaling Stations at South Georgia: a Study in Antartic Industrial Archaeology. Novus Fotrlag, Oslo.

Berríos, V. 2010. Recuerdo y olvido como parte de una historia: la Ballenera de Quintay. Memoria para optar al título de Antropóloga Social, Departamento de Antropología, Universidad de Chile, Santiago.

Brown, S.G. 1976. "Modern whaling in Britain and the 
north-east Atlantic Ocean”.MammalReview 6 (I): 25-36.

Coloane, F. 1972. Los balleneros de Quintay. Quimantú, Santiago.

Coloane, F. 2002. Tierra del Fuego. Alfaguara, Santiago.

Davis, L.E., R.E. Gallman y K. Gleiter. 1997. In Pursuit of Leviathan:Technology, Institutions, Productivity and Profits in American Whaling, I816-1906. The University of Chicago Press, Chicago.

De la Fuente, P. y D. Quiroz. 20I I. “Los chilotes en la ballenera de Quintay”. Revista Chilena de Antropología 24 (2): I7I-I9I.

García Márquez, G. 200I. Sofismas de distracción. Sala de Prensa, III (2). En www.saladeprensa.org/art20I.htm; consultado el 10 de julio de 2013.

Hohman, E. P. 1935. "American and Norwegian whaling: a comparative study of labor and industrial organization". The Journal of Political economy 43 (5): 628-652.

Compañía Industrial S.A. 1938. La Industria Ballenera. Compañía Industrial S.A.,Valparaíso.

Pastene, L.A. y D. Quiroz. 20I0. "Outline of the history of whaling in Chile. En Human Culture from the Perspective of Traditional Maritime Communities, International Symposium Report No. I, editado por International Center for Folk Culture Studies, Pp. 73-98. Kanagawa Shimbun Press, Kanagawa.

Pina Cabral, P. 2000. "The ethnographic present revisited”. Social Anthropology (3): 34I-348.

Quiroz, D. y P. de la Fuente. 2012. "Operaciones balleneras de la INDUS en aguas Patagónicas: años iniciales (19361939)". Magallania 40 (2): 23-40.

Reeves, R.R. y Smith, T.D. 2006."A taxonomy of world whaling: operations, eras, and data sources”. En Whales, Whaling and Ecosystems, editado por J.A. Estes, D.P. DeMaster, D.F. Doak, T.M.Williams y R.L. Brownell, Pp. 82-I0I. University of California Press, Berkeley.

Salazar, M. y J.C. Inostroza. 1997. Quintay en el Tiempo. Universidad Nacional Andrés Bello, Santiago.

Sanjek, R. 1991. "The ethnographic present". Man (n.s.) 26(4): 609-628

Thomas, K. 2009. The Ends of Life: Roads to Fulfillment in Early Modern England. Oxford University Press, Oxford

Tilly, Ch. 1978. "Anthropology, History and the Annales". Reviewl (3/4): 207-2I3.

Tønnesen, J.N. y A.O. Johnsen. 1982. The History of Modern Whaling. University of California Press, Berkeley and Los Angeles.

Reportajes

Anónimo.1943. “La caleta de Quintay será convertida en una planta para beneficiar ballenas”. Mar 100: 21 I-213.

Anónimo. 1953. “Sheblow off Chile”. Male 3(4): 40-43, 94-95.

Gauche, G. 1966. La caza de la ballena. El Mercurio (Valparaíso), I3 de marzo de 1966.

CI. 1954. Hvalfangst ved chilenske kyst.Norsk Hvalfangst Tidende 43 (5): I75- 180.

Montandón, R. 1949. Quintay, un barco fábrica anclado en un peñón. En Viaje 189:81.

Tischer, H. 1957. Das Ungeheuermit den Ölkopf. Constanze 10: 37, 92-94, 96-97.
Winet, G. 1943. En Quintay ha surgido la más moderna base ballenera del continente. ZigZag (Santiago), 3 de diciembre de 1943, pp. I5-16.

\section{Entrevistas}

Isaías Jaramillo, Quintay, 2008 [Entrevistó Paula de la Fuente]. José Barrios, Quintay, 2008 [Entrevistó Paula de la Fuente]. Fuente].

Isaías Jaramillo, Villa Alemana, 2010 [Entrevistó Paula de la

Daniel Barrios, Quintay, 2008 [Entrevistó Paula de la Fuente]. Alfredo Marín, Quintay 2009 [Entrevistó Violeta Berríos]. José Barrios, Quintay, 2009 [Entrevistó Violeta Berríos]. 